\title{
NEW DATA ABOUT THE COSTA RICAN APHID FAUNA (HEMIPTERA, APHIDIDAE)
}

\author{
D. Zamora Mejías ${ }^{1}$, N. Pérez Hidalgo ${ }^{2}$, W. Villalobos ${ }^{3}$ \& P. Hanson ${ }^{1}$
}

\begin{abstract}
D. Zamora Mejías, N. Pérez Hidalgo, W. Villalobos \& P. Hanson. 2012. New data about the Costa Rican aphid fauna (Hemiptera, Aphididae). Graellsia, 68(2): 305-312.

Aphids were collected from 39 locations throughout Costa Rica, representing a wide altitudinal range, and from both crops and native plants. In total, 48 species of aphids were identified, associated with 62 families and 111 species of plants, many of these representing new host plant records. Aphis nasturtii Kaltenbach, 1843 is reported here for the first time in Central America and Trichosiphonaphis (Xenomyzus) polygoni (van der Goot, 1917) for the first time in Costa Rica. This brings the total number of aphid species known from the country to 91 , representing $77,8 \%$ of all the known species in Central America.
\end{abstract}

Key words: Costa Rica; Aphis nasturtii; Trichosiphonaphis (Xenomyzus) polygoni; Costa Rica; aphids.

\section{RESUMEN}

D. Zamora Mejías, N. Pérez Hidalgo, W. Villalobos \& P. Hanson. 2012. Nuevos datos acerca de la fauna de áfidos de Costa Rica (Hemiptera, Aphididae). Grael/sia, 68(2): 305-312 (en inglés).

Los áfidos fueron colectados en 39 diferentes localidades en Costa Rica, sobre plantas nativas y cultivos, abarcando un amplio ámbito de altitud. En total, 48 especies de áfidos fueron identificadas, asociadas a 62 familias y 111 especies de plantas, varias representan nuevas asociaciones áfido-planta. Aphis nasturtii Kaltenbach, 1843 se cita por primera vez para América Central y Trichosiphonaphis (Xenomyzus) polygoni (van der Goot, 1917) por primera vez para Costa Rica. El total de especies de áfidos conocidas dentro del país se aumenta a 91, representando el 77,8\% de todas las especies citadas en América Central.

Palabras claves: Costa Rica; Aphis nasturtii; Trichosiphonaphis (Xenomyzus) polygoni; Costa Rica; áfidos.

Laboratorio Entomología, Escuela de Biología, Universidad de Costa Rica, A.P.2060, San Pedro de Montes de Oca, San José, Costa Rica-dazamoram@gmail

Departamento de Biodiversidad y Gestión Ambiental, Universidad de León, E-24071, León, España - nperh@unileon.es

3 Centro de Investigación en Biología Celular y Molecular, Universidad de Costa Rica, 11501-2060, San José, Costa Rica williamvillalobosm@gmail.com

Corresponding Author: Nicolás Pérez Hidalgo; E-mail: nperh@unileon.es 


\section{Introduction}

A large proportion of the aphids occurring in Central America are a result of human activities and natural invasions from the northern and southern hemispheres (Ortego et al., 2004; Villalobos Muller et al., 2010). Many species have arrived via introduced crops, ornamental plants and nursery seedlings, thereby increasing the number of aphid species present in the region (e.g. Pérez Hidalgo et al., 2009). In tropical regions aphid diversity increases with altitude (Eastop, 1998) and in mountainous countries such as Costa Rica a wide range of altitudes results in a diversity of microclimates, which in turn facilitates the successful establishment of new aphid species (Pérez Hidalgo et al., 2012). The study of this ever-changing aphid fauna is essential, since it contributes to our knowledge of their distribution and taxonomy, as well as strategies for the management and control of pest species (Ortego et al., 2004).

Work on the Central American aphid fauna began in the seventies and eighties (Holman, 1974; Smith \& Cermeli, 1979; Saunders et al., 1983) and in recent years research has increased considerably (Sánchez et al., 2001; Etienne, 2005; Evans \& Halbert, 2007; Quirós et al., 2009; Perez Hidalgo et al., 2009; Zamora Mejías et al., 2010; Villalobos Muller et al., 2010; Perez Hidalgo et al., 2012). Nonetheless, there is still much to be learned about the distribution and trophic relationships of the aphid species occurring in Central America. Costa Rica is one of the countries in which knowledge of aphids has increased significantly; as of early 2002 there were relatively few aphid species cited from the country and these were mainly from crops (Calvo, 1978; Chacón, 1980; Gómez, 1987; Hernández \& Meneses, 1988; Meneses \& Amador, 1987; Meneses \& Amador, 1990; Meneses et al., 1990; Rivera et al., 1994; Sánchez et al., 2001), but since the publication of the first list of aphids in the country (Voegtlin et al., 2003) sampling has increased and regular updates have appeared (Pérez Hidalgo et al., 2009; Zamora Mejías et al. 2010; Villalobos Muller et al. 2010; SánchezMonge et al., 2010). The recent publications have treated not only aphids of agricultural crops (which are often more susceptible to infestation), but also non-crop trees, shrubs, herbaceous plants and bryophytes.

Among the goals and challenges in the coming years of research on tropical aphids is the genera- tion of more information about their diversity, behavior and trophic relationships. Thus, the aim of the present study is to undertake a comprehensive survey of aphids in different locations in Costa Rica, identify the aphids and their host plants, and begin documenting the distribution of aphid species.

\section{Materials and methods}

The sampling was undertaken during 2008 and 2009 in several locations at different elevations in Costa Rica (Table 1), and these locations included both croplands and protected areas. Geographic data were taken using a GPS Garmin Etrex.

Aphid sampling was done by direct examination of plants, and aphid host plants were pressed and dried for later identification. Fern identifications were done by Alexander Rojas (Universidad Nacional de Costa Rica) and the remaining plants were identified by Jeffrey Vásquez and Jorge Gómez-Laurito (Universidad de Costa Rica); plant names are based on those used in the Manual of Costa Rican Plants (Hammel et al., 2003, 2004, 2007, 2010).

Pieces of host plants harboring aphid colonies were placed in plastic boxes $10 \mathrm{~cm}$ long on each side and $10 \mathrm{~cm}$ in height, with a ventilation hole covered with nylon fabric. Adult wingless and winged aphids were extracted daily from the boxes, and the aphids were preserved in $70 \%$ ethanol.

Specimens from each sample were mounted in Canada balsam on microscope slides following the methodology described in Voegtlin et al. (2003). Blackman \& Eastop $(1994,2000,2006)$ and Voegtlin et al. (2003) were used to identify the aphids. Specimens in 70\% ethanol and on microscope slides are deposited in the Museum of Zoology at the University of Costa Rica (MZUCR cra) and in the Aphidological collection at the University of León, Spain (CRI)

\section{Results}

In this study 48 species of aphids were identified, associated with 111 species and 62 families of plants (Table 2). Most of these host plant records involve polyphagous or oligophagous aphid species. 
Table 1.- Sampling locations of aphids in Costa Rica.

Tabla 1.- Localidades de colecta de áfidos en Costa Rica.

\begin{tabular}{|c|c|c|c|}
\hline & Province & Location & ation in meters \\
\hline 1 & Alajuela & Grecia & 1963 \\
\hline 2 & Alajuela & La Garita & 857 \\
\hline 3 & Alajuela & Palmares & 1100 \\
\hline 4 & Alajuela & Poás & 1588 \\
\hline 5 & Alajuela & San Ramón & 1098 \\
\hline 7 & Alajuela & Zarcero & 1648 \\
\hline 8 & Alajuela & Volcán Arenal & 532 \\
\hline 9 & Cartago & Cervantes & 1513 \\
\hline 10 & Cartago & Coris & 1424 \\
\hline 11 & Cartago & Cot & 1895 \\
\hline 12 & Cartago & La Unión & 1843 \\
\hline 13 & Cartago & Oreamuno & 1946 \\
\hline 14 & Cartago & Paraíso & 1079 \\
\hline 15 & Cartago & Taras & 1598 \\
\hline 16 & Cartago & Tierra Blanca & 2380 \\
\hline 17 & Cartago & Turrialba & 680 \\
\hline 18 & Cartago & Volcán Irazú & 3203 \\
\hline 19 & Guanacaste & Cañas & 26 \\
\hline 20 & Guanacaste & La Cruz & 2 \\
\hline 21 & Guanacaste & Liberia & 133 \\
\hline 22 & Guanacaste & Tilarán & 548 \\
\hline 23 & Heredia & Cariblanco & 848 \\
\hline 24 & Heredia & San Miguel & 1165 \\
\hline 25 & Heredia & Sarapiquí & 172 \\
\hline 26 & Limón & Colorado & 86 \\
\hline 27 & Puntarenas & Buenos Aires & 150 \\
\hline 28 & Puntarenas & Coto Brus & 1311 \\
\hline 29 & Puntarenas & Esparza & 160 \\
\hline 30 & Puntarenas & Garabito & 60 \\
\hline 31 & Puntarenas & Monteverde & 1382 \\
\hline 32 & Puntarenas & Osa & 10 \\
\hline 33 & Puntarenas & Sardinal & 161 \\
\hline 34 & San José & Cerro Buena Vista & 3161 \\
\hline 35 & San José & Coronado & 1758 \\
\hline 36 & San José & Desamparados & 1199 \\
\hline 37 & San José & Montes de Oca & 1214 \\
\hline 38 & San José & Mora & 1016 \\
\hline 39 & San José & Pérez Zeledón & 1706 \\
\hline
\end{tabular}

\section{New RECORDS For CENTRAL AmERICA AND COSTA RicA}

Aphis nasturtii Kaltenbach is reported here for the first time in Central America and Trichosiphonaphis (Xenomyzus) polygoni (van der Goot) is a new record for Costa Rica. These species are discussed in greater detail below, including the locality where they were collected, the geographic coordinates, elevation (in meters above sea level), date and lot number.
Aphis nasturtii Kaltenbach, 1843 (Aphidinae: Aphidini: Aphidina)

STUDIED MATERIAL: Apterous viviparous females were present on Xanthosoma mexicanum Liebm. (Araceae): Guanacaste province, Tilarán, $10^{\circ} 28.413^{\prime} \mathrm{N}, 84^{\circ} 57.961^{\prime} \mathrm{W}, 561 \mathrm{~m}, 17-\mathrm{IX}$ 09 (MZUCR cra 216; CRI-800); mixed with Pentalonia nigronervosa Coquerel.

They were collected on both sides of the leaves. The wingless females are pale green in life including the cauda and siphunculi. The legs and antennae show a slight dark pigmentation. The body is 0.9 to $2.4 \mathrm{~mm}$ in length. The antennae have 5-6 segments and are 0.4 to 0.8 times the body length. The terminal process of VI antennal joint is 1.2 to 3.5 times the length of the basal part. The apical rostral segment is 0.08 to $0.12 \mathrm{~mm}$ long the length of the second posterior tarsal segment, and has 1-3 secondary setae. The siphunculi are 0.13 to $0.30 \mathrm{~mm}$ in length. The abdomen lacks pigmentation, except for the stigmatic sclerites.

Aphis nasturtii is a species mentioned as present mainly in the Palearctic; in America it has been cited in Chile, Argentina, Canada, USA and Mexico (Blackman \& Eastop, 2006). It is cited as holocyclic on Rhamnus sp. (Rhamnaceae) and polyphagous on its secondary host plants, where it may sometimes be anholocyclic (Blackman \& Eastop, 2006), a phenomenon that may be occurring in Costa Rica.

Trichosiphonaphis (Xenomyzus) polygoni (van der Goot, 1917) (Aphidinae: Macrosiphini)

STUdied Material: Polygonum acuminatum Kunth (Polygonaceae): San José province, Herradura, Rivas, Pérez Zeledón, $9^{\circ}$ $30.057^{\prime} \mathrm{N}, 84^{\circ} 36.817^{\prime} \mathrm{W}, 1721 \mathrm{~m}, 20-\mathrm{IX}-09$ (MZUCR cra 154; CRI-771).

Wingless and winged females are yellow or dark green, sometimes almost black. The body length is 1.3-2.2 $\mathrm{mm}$. Secondary sensoria are present on antennal segments III, IV, V (20-41, 8-21, and 1-11, respectively), and the antennal tubercles are well developed. The cauda is long, without constrictions at the base, and has 4-15 hairs. The basal diameter of the siphunculi is at least 0.5 times the apical diameter.

Trichosiphonaphis (Xenomyzus) polygoni lives on Polygonum spp. in E. Asia (Japan, China, Taiwan, India, Pakistan and Java) and introduced in the USA (Smith \& Denmark, 1982) and Panama (Remaudière et al., 1992), but its life cycle is unknown (Blackman \& Eastop, 2006). 
Table 2.- New host plant records of aphids. For locality codes see Table $1 ;{ }^{*}$ indicates a new host plant record for the world (based primarily on Blackman \& Eastop, 1994, 2000, 2006); **indicates a new host plant record for Costa Rica.

Tabla 2.- Nuevas citas de plantas hospedadoras. Los códigos de localidades se encuentran en la Tabla 1; * indica una nueva cita de planta hospedadora para el mundo (basado principalmente en Blackman \& Eastop, 1994, 2000, 2006); ${ }^{\star *}$ indica un nuevo reporte de planta hospedadora para Costa Rica.

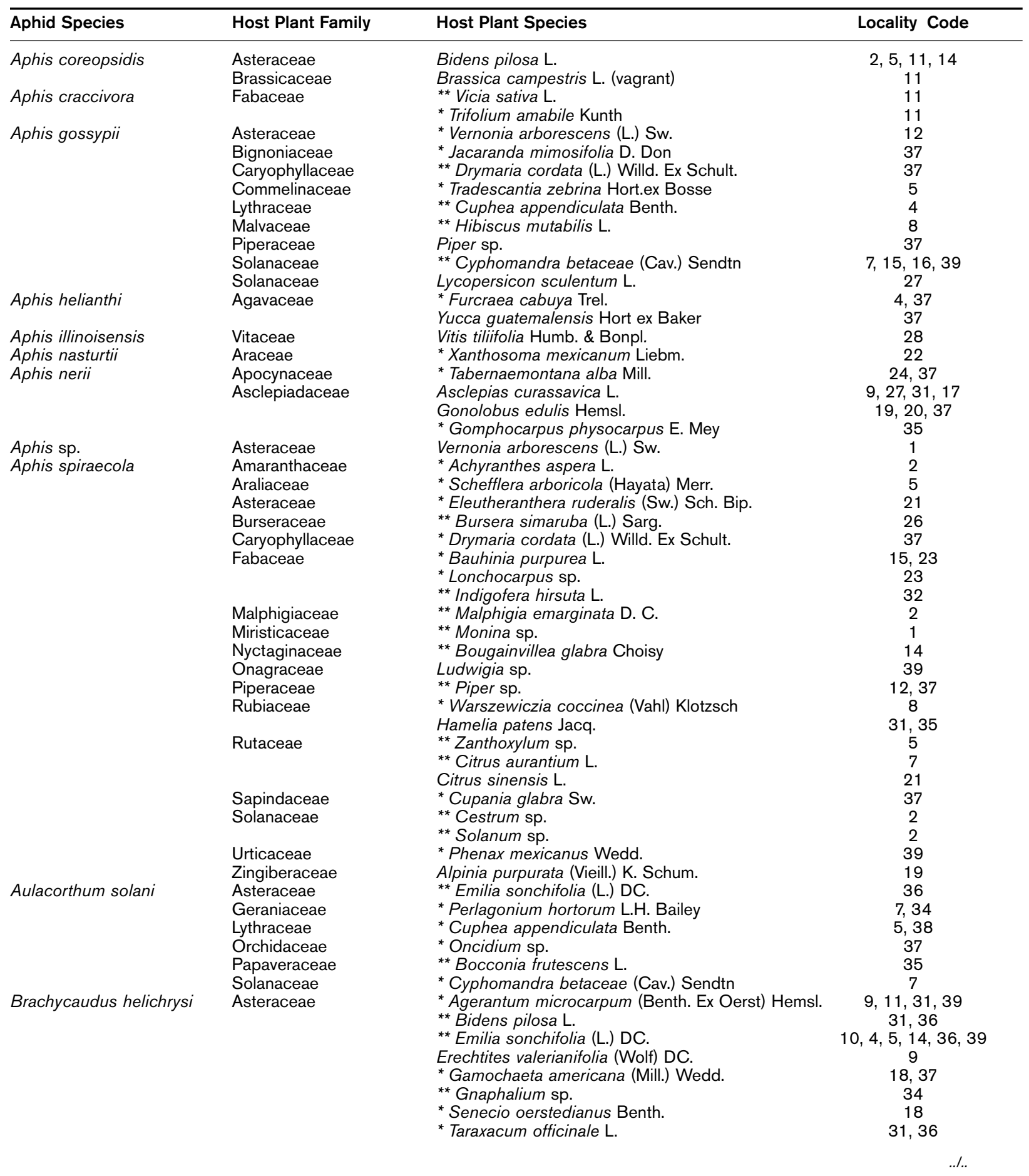


Table 2.- Cont.

\begin{tabular}{|c|c|c|c|}
\hline Aphid Species & Host Plant Family & Host Plant Species & Locality Code \\
\hline & & Unknow host plant & 34 \\
\hline & Cyperaceae & Cyperus hermaphroditus (Jacq.) Standl. & 39 \\
\hline & Loasaceae & * Nasa triphylla Juss. & 35 \\
\hline & Lythraceae & * Cuphea appendiculata Benth. & 5 \\
\hline & Ranunculaceae & ** Ranunculus repens $\mathrm{L}$. & 35 \\
\hline & Solanaceae & Solanum sp. & 34 \\
\hline & Valeraniaceae & * Valeriana prionophylla Standl. & 34 \\
\hline \multirow{2}{*}{$\begin{array}{l}\text { Brevicoryne brassicae } \\
\text { Cerataphis brasiliensis }\end{array}$} & Brassicaceae & ** Brassica campestris L. & $9,10,11$ \\
\hline & Arecaceae & $\begin{array}{l}\text { * Washingtonia filifera (Linden) H.Wenc } \\
\text { Chamaedorea costaricana Oerst }\end{array}$ & $\begin{array}{l}5,26 \\
37\end{array}$ \\
\hline \multirow{2}{*}{ Cerataphis orchidearum } & Orchidaceae & ${ }^{*}$ Cattleya skinneri Bateman & 5 \\
\hline & Zingiberaceae & Alpinia purpurata (Vieill.) K. Schum. & 30 \\
\hline $\begin{array}{l}\text { Cinara fresai } \\
\text { Cinara watsoni }\end{array}$ & $\begin{array}{l}\text { Cupressaceae } \\
\text { Pinaceae }\end{array}$ & $\begin{array}{l}\text { ** Cupresus lusitanica Mill. } \\
\text { * Pinus devoniana Lindl. }\end{array}$ & $\begin{array}{c}1,7,16,34,35 \\
34\end{array}$ \\
\hline $\begin{array}{l}\text { CInara watsonI } \\
\text { Greenidea psidii }\end{array}$ & $\begin{array}{l}\text { PInaceae } \\
\text { Myrtaceae }\end{array}$ & $\begin{array}{l}\text { PInus devoniana LIndl. } \\
\text { Psidium guajava L. }\end{array}$ & $1,5,14,22,23,28,31$ \\
\hline Hyperomyzus caduellinus & Asteraceae & * Taraxacum officinale L. & 9,37 \\
\hline \multirow[t]{3}{*}{ Hysteroneura setariae } & Cyperaceae & ${ }^{*}$ Rhynchospora polyphylla Valh & 37 \\
\hline & Poaceae & ** Pennisetum purpureum Schumach. & 33 \\
\hline & & $\begin{array}{l}{ }^{* *} \text { Paspalum sp. } \\
{ }^{* *} \text { Cynodon nlemfuensis Vanderyst }\end{array}$ & $\begin{array}{l}37 \\
37\end{array}$ \\
\hline \multirow{7}{*}{ Idiopterus nephrelepidis } & Blechnaceae & Blechnum schiedeanum (Schltd. ex) C. Pres H & 35 \\
\hline & Lomariopsidaceae & Nephrolepis cordifolia (L.)C. Presl & 23 \\
\hline & Cupressaceae & Cupresus lusitanica Mill. & 35 \\
\hline & Brassicaceae & ${ }^{* *}$ Brassica campestris L. & 34 \\
\hline & & Brassica oleraceae L. & 39 \\
\hline & Unknown family & Unknown host plant & 29 \\
\hline & Loasaceae & * Nasa triphylla Juss. & 35 \\
\hline \multirow{6}{*}{$\begin{array}{l}\text { Lizerius sp. } \\
\text { Macrosiphoniella sanborni } \\
\text { Macrosiphum euphorbiae } \\
\text { Macrosiphum rosae } \\
\text { Macrosiphum salviae }\end{array}$} & Lauraceae & ** Ocotea sp. & 2 \\
\hline & Asteraceae & Chrysanthemum sp. & 37 \\
\hline & Solanaceae & Lycopersicum sculentum L. & 13 \\
\hline & Rosaceae & ${ }^{* *}$ Rosa chinensis Jacq. & 34,37 \\
\hline & Lamiaceae & ${ }^{* *}$ Ocimum sanctum $\mathrm{L}$. & 2 \\
\hline & Myricaceae & ${ }^{*}$ Morella pubescens (Willd.) Wilbur & 34 \\
\hline \multirow{16}{*}{$\begin{array}{l}\text { Microparsus pojanii } \\
\text { Myzodium modestum } \\
\text { Myzus ascalonicus } \\
\text { Myzus hemerocallis } \\
\text { Myzus ornatus }\end{array}$} & Polypodiaceae & * Phlebodium pseudoaureum (Cav.) Lellinger & 11 \\
\hline & Polytrichaceae & ** Polytrichum juniperinum Hedw. & 34 \\
\hline & Ranunculaceae & ${ }^{* *}$ Ranunculus repens $\mathrm{L}$. & 35 \\
\hline & Liliacae & ${ }^{* *}$ Hemerocallis fulva (L.) L. & 37 \\
\hline & Araliaceae & * Oreopanax pycnocarpus Donn. Sm. & 34 \\
\hline & Asteraceae & Unknow host plant & 37 \\
\hline & & * Gamochaeta americana (Mill.) Wedd. & 37 \\
\hline & Lamiaceae & Salvia sp. & 34 \\
\hline & Polygonaceae & Rumex sp. & 34 \\
\hline & Ranunculaceae & ${ }^{*}$ Ranunculus repens $\mathrm{L}$. & 35 \\
\hline & Rosaceae & * Rubus urticifolius Poir. & 34 \\
\hline & Rutaceae & ${ }^{* *}$ Citrus sinensis $\mathrm{L}$. & 4 \\
\hline & Scrophulariaceae & * Digitalis purpurea L. & 34 \\
\hline & Solanaceae & ** Cyphomandra betaceae (Cav.) Sendtn & 9,15 \\
\hline & & Solanum sp. & 34 \\
\hline & Valeraniaceae & * Valeriana prionophylla Standl. & 34 \\
\hline \multirow[t]{8}{*}{ Myzus persicae } & Apocynaceae & Catharanthus roseus (L.) G. Don. & 37 \\
\hline & Asteraceae & ${ }^{* *}$ Bidens pilosa L. & 36 \\
\hline & Brassicaceae & ${ }^{* *}$ Brassica campestris N/D & 10,11 \\
\hline & Caryophyllaceae & ${ }^{* *}$ Drymaria cordata (L.) Willd. Ex Schult. & 37 \\
\hline & Loasaceae & *Nasa triphylla Juss. & 35 \\
\hline & Lythraceae & ${ }^{*}$ Cuphea appendiculata Benth. & 4 \\
\hline & Polygonaceae & * Polygonum acuminatum Kunth & 35 \\
\hline & Solanaceae & Lycopersicum esculentum L. & 7 \\
\hline \multirow[t]{2}{*}{ Neomyzus circumflexum } & Fabaceae & * Trifolium amabile Kunth & 11 \\
\hline & Rubiaceae & ** Hamelia patens Jacq. & 35 \\
\hline \multirow{7}{*}{$\begin{array}{l}\text { Neophyllaphis araucariae } \\
\text { Pentalonia nigronervosa }\end{array}$} & Araucariaceae & * Araucaria excelsa (Lamb.) R. Br & 23,28 \\
\hline & Araceae & * Xanthosoma mexicanum Liebm. & 22 \\
\hline & Costaceae & * Costus pulverulentus C. Presl. & 37 \\
\hline & Heliconiaceae & * Heliconia rostrata Ruiz \& Pavón & 3,25 \\
\hline & Musaceae & ${ }^{*}$ Musa acuminata $x$ balbisiana & 21 \\
\hline & Zingiberaceae & ${ }^{\star *}$ Elettaria cardomomum (L.) Maton & 5 \\
\hline & & ${ }^{* *}$ Alpinia purpurata (Vieill.) K. Schum. & $22,25,30$ \\
\hline Rhodobium porosum & Solanaceae & Solanum sp. & 34 \\
\hline
\end{tabular}


Table 2.- Cont.

\begin{tabular}{|c|c|c|c|}
\hline Aphid Species & Host Plant Family & Host Plant Species & Locality Code \\
\hline Rhopalosiphum maidis & Poaceae & Zea mays L. & 11,39 \\
\hline Schizaphis rotundiventris & Cyperaceae & ${ }^{\star}$ Cyperus odoratus $\mathrm{L}$. & 13 \\
\hline \multirow[t]{3}{*}{ Sipha flava } & Cyperaceae & * Cyperus odoratus L. & 13 \\
\hline & Polygonaceae & Rumex sp. & 13 \\
\hline & Rosaceae & Rubus urticifolius Poir. (vagrant) & 34 \\
\hline Sitobion luteum & Orchidaceae & * Oerstedella schummaniana & 8 \\
\hline Sarucallis kahawaluokalani & Myrtaceae & * Eugenia uniflora L. & 37 \\
\hline \multirow[t]{13}{*}{ Toxoptera aurantii } & Anacardiaceae & * Spondias purpurea L. & 31 \\
\hline & Chrysobalanaceae & ${ }^{*}$ Licania platypus (Hemls.) Fritsch & 2 \\
\hline & Clusiaceae & ${ }^{*}$ Calophyllum inophyllum L. & 26 \\
\hline & Ericaceae & * Cavendishia sp. & 11 \\
\hline & & * Cavendishia bracteata Ruíz \& Pav. ex J.St.-Hi Hoerold & 34 \\
\hline & Lythraceae & * Cuphea appendiculata Benth. & 38,39 \\
\hline & Myrtaceae & * Psidium friedrichsthalianum (O. Berg) Nied. & 5 \\
\hline & Passifloraceae & ${ }^{* *}$ Passiflora edulis Sims & 1 \\
\hline & Polygonaceae & ** Coccoloba sp. & 26 \\
\hline & Rutaceae & Citrus sinensis L. & 26 \\
\hline & Sapindaceae & * Litchi chinensis Sonn. & 2 \\
\hline & Solanaceae & ${ }^{* *}$ Cestrum sp. & 26 \\
\hline & Winteraceae & * Drymis granadensis L. & 34 \\
\hline \multirow[t]{5}{*}{ Toxoptera citricidus } & Myrcinaceae & * Ardisia sp. & 5 \\
\hline & Rubiaceae & * Hamelia patens Jacq. & 25 \\
\hline & Rutaceae & ${ }^{* *}$ Citrus aurantium L. & 5 \\
\hline & & Citrus sinensis $\mathrm{L}$. & $4,5,25,30,37$ \\
\hline & & * Zanthoxylum sp. & 5 \\
\hline Irıchosiphonaphis polygonı & Polygonaceae & * Polygonum acuminatum Kunth & 39 \\
\hline \multirow{3}{*}{ Uroleucon ambrosiae } & Salicaceae & ** Salix humboldtiana Willd. & 39 \\
\hline & Asteraceae & $\begin{array}{l}\text { * Agerantum microcarpum (Benth. Ex Oerst) Hemsl. } \\
\text { * Emilia sonchifolia (L.)DC. } \\
\text { Verbesina gigantea Jacg. }\end{array}$ & $\begin{array}{c}39 \\
5 \\
438\end{array}$ \\
\hline & Unknown family & $\begin{array}{l}\text { Verbesina gigantea Jacq. } \\
\text { Unknown host plant }\end{array}$ & $\begin{array}{l}4,38 \\
37\end{array}$ \\
\hline \multirow[t]{2}{*}{ Uroleucon gravicorne } & Asteraceae & Conyza canadensis (L.) Cronquist & 4,37 \\
\hline & Rosaceae & Rubus sp. & 34 \\
\hline
\end{tabular}

The genus Trichosiphonaphis is closely related to Myzus and its origin is mainly in the Palaeartic and East Asia, where it alternates between Lonicera (Caprifoliaceae) and Polygonum (Polygonaceae) (Blackman \& Eastop, 2006). Remaudière et al. (1992) provided a review of the genus and a key to the species.

\section{Discussion}

As of 2010 the number of aphid species (Aphididae) cited from Costa Rica was 88 (Villalobos Muller et al., 2010) and later this number increased to 89 (Pérez Hidalgo et al., 2012). With the new records from the present study the number is now 91 species, compared with a total of 62 species reported by Voegtlin et al. (2003), and representing 77,8\% of the 117 species known in Central America. This is the highest number of aphid species reported from any country or territo- ry in Central America and the Caribbean, surpassing the 71 species in Panama (Quirós et al., 2009), 26 in Nicaragua (Smith \& Cermeli, 1979; Saunders et al., 1983), 44 in Honduras (Evans \& Halbert, 2007), 82 in Cuba (Holman, 1974; Etienne 2005), 41 in Puerto Rico (Étienne, 2005), and 58 in Guadeloupe (Etienne, 2005).

The species that were most frequently collected during this study were Aphis gossypii Glover, Aphis spiraecola Patch, Myzus ornatus Laing, Toxoptera aurantii (Boyer de Fonscolombe) and Toxoptera citricidus (Kirkaldy), which indicates a stable and extensive presence in the country. Other species that were also frequently collected were Aphis coreopsidis (Thomas), Aulacorthum solani (Kaltenbach), Brachycaudus helichrysi (Kaltenbach), Myzus persicae (Sulzer) and Hysteroneura setariae (Thomas) which, like the above species, are not native to Central America (Cermeli, 2006), but are now widely distributed and present on a wide range of host plant species. 
Aphid-plant relationships are of vital importance when studying aphid population dynamics and how they might modify the dynamics of plant communities. Among the aphid-plant associations that deserve special attention are some that were previously known and which directly impact crops throughout the country. Aphis spiraecola and Toxoptera citricidus were frequently collected, mainly on Citrus, although the latter aphid is highly polyphagous (Voegtlin et al., 2003; Villalobos Muller et al., 2010). Greenidea psidii van der Goot has been widely distributed, especially on plants in the family Myrtaceae (Pérez Hidalgo et al., 2009). Toxoptera aurantii is also widely distributed throughout the country (Voegtlin et al., 2003; Villalobos Muller et al., 2010) and it feeds on many native plants (Table 2 ), which suggests that it has adapted to natural ecosystems; for example, Coccoloba uvifera L., a common plant on both coasts (Zuchowski, 2005), was sometimes highly infested. Pentalonia nigronervosa (Coquerel), originally on Musaceae, is also found on Zingiberaceae and Heliconiaceae in Costa Rica, both of which are native families with species of economic importance as ornamentals (Voegtlin et al., 2003; Villalobos Muller et al., 2010). Cerataphis brasiliensis (Hempel) on palms and Cerataphis orchidearum (Westwood) on orchids also affect ornamental plants, as do Brachycaudus helichrysi and the polyphagous species comprising the Aphis and Uroleucon complexes.

While some aphid species are restricted to just a few species of host plants, many are both crop pests and present in native ecosystems. The less frequently collected species, including the new records presented here, can potentially provide information that is useful in the monitoring and management of aphids in the country. More research is needed on the aphids of native plants in order to identify the native species (undoubtedly a minority of the aphid fauna), how the introduced species become adapted and how they might affect native plants, and whether some native plants provide reservoirs for crop pests.

\section{Acknowledgements}

We thank the University of Costa Rica, the Agency for International Development Cooperation (AECID) (ref. $\mathrm{D} / 010523 / 07$ ) and Idea Wild for financial support.

\section{References}

Blackman, R. L. \& Eastop, V. F., 1994. Aphids on the world's trees. An identification and information guide. CAB International. Oxon. 986 pp.

Blackman, R. L. \& Eastop, V. F., 2000. Aphids on the world's crops. An identification and information guide. John Willey \& Sons. Chichester. 466 pp.

Blackman, R. L. \& Eastop, V. F., 2006. Aphids on the world's herbaceous plants and shrubs. Wiley. Chichester. 1439 pp.

Calvo, C., 1978. Variación estacional del áfido Myzus persicae (Sulzer) en la Estación Experimental Agrícola Fabio Baudrit. Maestría Tesis Ingeniería Agrícola. Universidad de Costa Rica, Facultad de Agronomía. San José. 27 pp.

Cermeli, M., 2006. Diversidad y origen de la afidofauna venezolana (Hemiptera: Aphidoidea). Entomotropica, 21(2): 83-89.

Chacón, C., 1980. Evaluación de la población de áfidos alados en cultivos de papa para semilla en la zona norte de Cartago. Tesis Ingeniería Agrícola. Universidad de Costa Rica, Facultad de Agronomía. San José. 80 pp.

Eastop, V. F., 1998. Why do aphids do that? In: J.M. Nieto Nafría \& A.F.G. Dixon (eds.), Aphids in natural and managed ecosystems. Universidad de León (Secretariado de Publicaciones). León: 37-47.

Étienne, J., 2005. Les pucerons de Guadeloupe, des Grandes et Petites Antilles (Hemiptera, Aphididae). Bulletin de la Société Entomologique de France, 110: 455-462.

Evans, G. A. \& Halbert, S. E., 2007. A checklist of the aphids of Honduras (Hemiptera: Aphididae). Florida Entomologist, 90: 518-523.

Gómez, C., 1987. Fluctuación de la población de áfidos $e$ incidencia de virus " $Y$ " en tabaco en el cantón de Pérez Zeledón, provincia de San José, Costa Rica. Tesis Ingeniería Agrícola. Universidad de Costa Rica, Facultad de Agronomía. San José. 80 pp.

Hammel, B. E., Grayum, M. H., Herrera, C. \& Zamora, N., 2003, 2004, 2007, 2010. Manual de plantas de Costa Rica. VI Volúmenes. Missouri Botanical Garden Press. St. Louis. 931 pp.

Hernández, J. \& Meneses, R., 1988. Nota descriptiva del pulgón lanígero (Eriosoma lanigerum) de la manzana en Costa Rica. Manejo Integrado de Plagas, Revista del Proyecto MIP/CATIE, 8: 22-26.

Holman, J., 1974. Los áfidos de Cuba. Instituto Cubano del Libro. La Habana. 304 pp.

Meneses, R. \& Amador, R., 1987. Evaluación preliminar de la fluctuación de áfidos en la zona norte de Cartago, Costa Rica. Manejo Integrado de Plagas, Revista del Proyecto MIP/CATIE, 5: 16-20. 
Meneses, R. \& Amador, R., 1990. Los áfidos alados de la papa y su fluctuación en Costa Rica. Manejo Integrado de Plagas, Revista del Proyecto MIP/CATIE, 15: 3544.

Meneses, R., Ramírez, A. \& Piaggio, G., 1990. Efectos de tres tipos de trampas de agua en la captura de áfidos. Manejo Integrado de Plagas, Revista del Proyecto MIP/CATIE, 18: 13-18.

Ortego, J., Difabio, M. E. \& Mier Durante M. P., 2004. Nuevos registros y actualización de la lista faunística de los pulgones (Hemiptera: Aphididae) de La Argentina. Revista de la Sociedad Entomológica Argentina, 63: 19-30.

Pérez Hidalgo, N., Villalobos Muller, W. \& Mier Durante, M. P., 2009. Greenidea psidii (Hemiptera: Aphididae: Greenideinae) new invasive aphid in Costa Rica. Florida Entomologist, 92: 396-398.

Pérez Hidalgo N., Martínez-Torres, D., Collantes-Alegre, J. M., Villalobos Muller, W. \& Nieto Nafría J. M., 2012. A new species of Rhopalosiphum (Hemiptera, Aphididae) on Chusquea tomentosa (Poaceae, Bambusoideae) from Costa Rica. ZooKeys, 166: 5973.

Quirós, D. I., Remaudière, G. \& Nieto Nafría, J. M., 2009. Contribución al conocimiento de Aphididae y Phylloxeridae (Hemiptera, Sternorrhyncha) de Panamá. Neotropical Entomology, 38: 791-800.

Rivera, C., Villalobos, W., Sánchez, M. V., Zumbado, C. \& Rodríguez C. M., 1994. Identification and distribution of melon-infesting viruses and their vectors in two provinces of Costa Rica. Turrialba, 43: 210-215.

Remaudière, G., Serain, M., Trouvé, C. \& Demeester, S., 1992. Données nouvelles sur le genre Trichosiphonaphis. Revue Francaise d'Entomologie, 14: 49-58.

Sánchez, M. V., Agüero, R. \& Rivera, C., 2001. Plantas hospederas de Aphis gossypii (Aphididae), vector del virus del melón Cucumis melo (Cucurbitaceae) en Costa Rica. Revista de Biología Tropical, 49: 305210.

Sánchez-Monge, A., Retana-Salazar, A., Brenes, S. \& Agüero, R., 2010. New records of aphid-plant associations (Hemiptera: Aphididae) from eastern Costa Rica. Florida Entomologist, 93: 489-492.

Saunders, J. L., King, A. B. \& Vargus, G. L., 1983. Plagas de cultivos en América Central: Una lista de referencia. Centro Agronómico Tropical de Investigación y Enseñanza, Departamento de Producción Vegetal, 9: 1-90.
Smith, C. F. \& Cermeli, M., 1979. An annotated list of Aphididae (Homoptera) of the Caribbean islands and South and Central America. North Carolina Agricultural Experiment Station Technical Bulletin, 259: 1-131.

Smith, C. F. \& Denmark, H. A., 1982. Trichosiphonaphis polygoni a genus and species new to the United States. Florida Entomologist, 65: 381-382.

Villalobos Muller, W., Pérez Hidalgo, N., Mier Durante, M. P. \& Nieto Nafría, J. M., 2010. Contribución al conocimiento de la fauna de pulgones (Hemiptera, Sternorrhyncha: Aphididae) de Costa Rica. Boletín de la Asociación Española de Entomología, 34: 145182.

Voegtlin, D., Villalobos, W., Sánchez, M. V., Saborío, G. \& Rivera C., 2003. Guía de los áfidos alados de Costa Rica. Revista Biología Tropical, 51: 1-228.

Zamora Mejías, D., Pérez Hidalgo, N. \& Mier Durante M. P., 2010. First report of Idiopterus nephrelepidis (Hemiptera: Aphididae) in Central America. Florida Entomologist, 93(3): 460-463.

Zuchowski, W., 2005. A guide to tropical plants of Costa Rica. Zona Tropical Publications. Miami. 529 pp. 